\title{
Calcineurin inhibitors and related medicines: a cohort study examining England's primary care prescription changes during the COVID-19 pandemic (January 2019 to March 2021)
}

\author{
Ravina Barrett ${ }^{1}$ (D)
}

Received: 10 September 2021 / Accepted: 22 December 2021 / Published online: 24 January 2022

(c) Springer Nature Switzerland AG 2022

\begin{abstract}
Purpose To examine the impact of the COVID-19 pandemic on calcineurin inhibitors and related prescriptions for community patients in England.

Methods Data from all primary-care patients who had calcineurin inhibitors prescriptions, dispensed in the community in England were included. Descriptive statistics and interrupted time series analysis over 27 months (15 months before and 12 months after $1^{\text {st }}$ lockdown) was evaluated.

Results Descriptive statistics show that mean values have declined since the pandemic's onset. Over the 27 months: mean Tacrolimus 865,045 doses, standard deviation (SD) 76,147 doses, with 95\% CI 834,923, 895,168, (min 567,508, max 1,010,900), ciclosporin 315,496 doses, SD 40,094, 95\% CI 299,635, 331,356 (min 191,281, max 382,253) and sirolimus $21,384$ doses, SD 2,610, 95\% CI 20,352, 22,417 ( $\min 13,022, \max 26,156)$. Analysis of variance between the pre- and post- periods show significant variations in quantities of tacrolimus $F 7.432, p=0.012$, ciclosporin $F 33.147, p<0.001$ and sirolimus F 18.596, p $<0.001$ (1df), mirrored in price analysis. The Interrupted Time Series (ARIMA Modelling) shows declining trends. After the pandemic's onset, a statistically significant downward trend in quantity for tacrolimus $\mathrm{p}=0.008$ is observed, with an estimated monthly decline of 14,524 doses, ciclosporin $p=0.185$, with an estimated decline of 2,161 doses and sirolimus $\mathrm{p}=0.002$ with an estimated decline of 485 doses, along with declining prices.

Conclusion A decrease in prescription medicines use raises concerns for the care of (renal) transplant patients. Patients are encouraged to discuss their planned care with their doctor, secure supplies and remain adherent to their medication.
\end{abstract}

Keywords Calcineurin inhibitors · Follow-up · Acute rejection · Calcineurin inhibitors · COVID-19 · Graft rejection · Tacrolimus $\cdot$ Ciclosporin $\cdot$ Sirolimus

Key Learning Points What is already known about this subject? - Tacrolimus, ciclosporin and sirolimus prevent transplant rejections, improves quality of life and are life sustaining for patients who have received a transplanted organ eg kidney. - However, these medicines should be prescribed and dispensed by brand because products are not bioequivalent and unsupervised switching between brands and formulations could lead to loss of rejection control, increased toxicity and risks of transplanted organ rejection.

What this study adds:

- Patients are not taking their calcineurin inhibitors and related medicines as intended.

- Findings suggest medicines use is declining significantly in the 12 months after the onset of the pandemic in England (Apr20Mar21 as compared to Jan19-Mar20), which is concerning for this vulnerable patient group.

\begin{abstract}
- Findings provide early warning signal for deteriorating care for this vulnerable group of patients who are more predisposed to infection risk including for COVID due to their immunocompromised status

What impact this may have on practice or policy:

- There is a potentially growing or accelerating unmet medical need in patients with the real and potential risk that this damages their health, may lead to organ rejections or higher rate of compilations or failures than previously expected/observed. - Five-year organ survival rates may fall below 93\% rate, which currently stands at riskadjusted percentages ranging from $88 \%$ to 97\% between national kidney transplant centres.
\end{abstract}

Extended author information available on the last page of the article 


\section{Introduction}

Calcineurin inhibitors and related medicines include tacrolimus, ciclosporin and sirolimus, which are highly potent immunosuppressive agents. They are routinely used in clinical practice for the prevention of organ rejection in kidney allograft recipients, amongst other kinds of transplants [1]. Tacrolimus and ciclosporin are also sometimes used within the management (as second-line therapy) of rheumatoid arthritis, ulcerative colitis, atopic dermatitis and when conventional therapy may be ineffective or inappropriate e.g. conventional synthetic Disease-modifying antirheumatic drugs (csDMARDs) use in Immune-Mediated Inflammatory Diseases (IMIDs) sufferers. As a result, these medicines have a vital role in the lives of transplant patients, where these medicines prevent transplant rejections, improves quality of life and are life sustaining.

Important regulatory and safety guidance for clinicians and pharmacists suggests that these medicines should be prescribed and dispensed by brand-name because switching between formulations and brands without close monitoring could lead to important clinical changes in blood plasma levels resulting in variable control and or toxicity[2]. Immediate release and prolonged release formulations are not interchangeable due to their differing bioavailability, pharmacokinetic and pharmacodynamic handling within patients. There are a variety of known adverse effects and interactions that further need careful monitoring alongside the routine blood monitoring that is necessary.

Kidney function needs to be monitored alongside lipid and urine protein levels to ensure that the adverse effects of drugs are not deleterious. A variety of national[3], international and regulatory via the Summary of Product Characteristic (SmPC) Commission on Human Medicines (CHM) guidance[2] provide evidence based up-to-date clinical guidance for a drug management therapies in this small, but extremely vulnerable patient population. In the United Kingdom (UK), costs are absorbed by the National Health Service (NHS) and clinical-economic decisions of funding and medicinal provisions are agreed with the commercial medicine's unit, indicating the need for cost containment, while providing patient benefits. Patients already stabilised on these therapies should not amend their therapy without careful monitoring, and usually in concert with their clinicians. High rates of adherence and compliance are important to the continued success of the transplant. Abrupt stopping, tapering or withdrawal should not be done [4]. Well-planned withdrawal should be under supervision with small monitoring intervals.

Immunosuppressants like calcineurin inhibitors can lead to opportunistic infections, including COVID-19 [5]. Early evidence suggests that proactive care may be necessary for this vulnerable patient group[6] with the need for avoiding Acute kidney injury (AKI) [7]. AKI is relatively common in critically ill COVID-19 patients and is known to increase mortality and prolong hospital stay [8, 9]. this long term need for self care, particularly becomes burdensome for patients who spend a long time on the transplant waiting list. Care within ethnic minority groups[10] can be difficult with patients being on the waiting list[11] for longer periods of time due to a shortage of organs from suitably appropriate ethnic minority donors, there may be other barriers such as language, cultural or misunderstanding of service[12] care pathways [13]. Some evidence suggests that young adults may have worse outcomes [14]. Many UK transplant centres currently provide restricted access due to COVID-19 [15]. There are early pandemic signals of reduced prescription use of such medicines [16]. Key dates of importance[17]: 23rd March 2020 (First lockdown), 31st October 2020 (Second lockdown), 5th January 2021 (Third lockdown) and Public vaccination began in January 2021 in England.

\section{Materials and methods}

The methods used in this study have been previously published in detail $[18,19]$.

\section{Study design}

This was a retrospective cohort study of all patients in England, within primary care who were prescribed calcineurin inhibitors and related medicines. The exposure was to the global pandemic. Prescription claims data in England before and after the pandemic's onset were compared. Statistical variations were the outcome of interest.

\section{Data source}

The 'English Prescribing Dataset' (EPD)[20] contains public sector information licensed under the Open Government Licence V2.0, which provides anonymised prescription data in England. ${ }^{24}$ The EPD contains detailed information on community-issued prescriptions (and another data set for hospital) issued in England, but which were dispensed across the UK (England, Wales, Scotland, Guernsey, Alderney, Jersey, and the Isle of Man). It contains detailed prescribing information at practice level, which are aggregated by British National Formulary (BNF) codes to maintain patient confidentiality. Therefore, each row of data is aggregated at practice level and does not represent individual patients or prescriptions. The data includes total quantity of 
unit-doses (e.g., pre-filled syringes, vials), and 'actual cost' for reimbursement.

The data excludes prescriptions issued outside England (Wales, Scotland, Guernsey, Alderney, Jersey, and the Isle of Man - this difference is solely to do with where prescriptions were issued as opposed to where dispensed); items not dispensed, disallowed and those returned for further clarification; those prescribed and dispensed in prisons, hospitals and private prescriptions; items prescribed but not presented for dispensing or not submitted to NHS prescription services by the dispenser. This dataset includes small operational irregularities (e.g., 17 rows in Jan 2019 of 'unidentified practice data', 470 rows of 'NULL' chemical substance codes where accurate BNF codes were given to permit extraction of the missing data). The study population represents English residents who were issued a prescription and had it dispensed.

Monthly data from January 2019 to March 2021, was examined for all calcineurin inhibitor medicines which included: tacrolimus, ciclosporin and sirolimus. Relevant rows of data were extracted using Structured Query Language (SQL). After excluding unnecessary rows, the data were filtered down to 36,792 relevant rows of data. Data extraction and cleaning methods have been previously reported [18]. Data were aggregated by month, chemical substance, regional office name and BNF code, to allow for human analysis.

The population was assumed to be constant as the study period maps the first lockdown where movement restrictions were in place. The target population cohorts consisted of patients prescribed and dispensed these medicines, but their exact diagnosis remains undocumented and therefore unknown. Lockdown commenced on 23rd of March 2020 with subsequent local and national lockdowns. The data source does not provide demographic or individual level data. Medicines shortages were explored via the national medicines' agency Central Alerting System (https://www. cas.mhra.gov.uk/SearchAlerts.aspx).

\section{Statistical analysis}

Analysis was carried out in Excel ${ }^{\circledR}$ v. 2007 and SPSS $®$ v. 26 (Supplementary 5 Syntax File). Results are presented as proportions, descriptive statistics, and hypothesis testing at $95 \%$ confidence level and by monetary value in pounds sterling.

An interrupted times series (ITS) design[21-23] was used. A commonly used time series Autoregressive integrated moving average (ARIMA) [24] modelling framework was employed to analyse the monthly total-quantity of prescription data from the EPD. ARIMA is a flexible modelling construct, allowing lagged correlations and seasonal differences to be modelled. ITS, employing ARIMA measures whether a natural event like the pandemic causes abrupt changes in the level or the pre-existing trend (slope) of study outcomes and is appropriate for examining the impact of natural events at a population level. Whether the pandemic was associated with a step change or a change in ITS trend since March 2020 was assessed. In practice, month-to-month patients may be issued a prescription but may receive the medication in the subsequent month, as a result, a one-month autoregressive component was applied to the model to represent this clinical practice (See Supplemental Sensitivity Analysis \& Syntax). The observed temporal trend in prescription total-quantity was explored by scatterplot in advance of performing the main time series analysis. Seasonal adjustments or lags were considered unnecessary because of the requirement to take these medications daily, year-round. 27 consecutive monthly data points with the interrupt time set at the $15^{\text {th }}$ month (March 2020) were available, and 15 data points before and 12 data points after were examined. The model detects differences in these two timeframes with respect to their linear trends i.e., the difference between the slope of the lines before and after March 2020 (intercept). A 10\% sample validation was done against an independent platform: https://openprescribing. net/. While every effort has been made to validate the data, it has not been possible to independently validate against manufacturers. This study followed the REporting of studies Conducted using Observational Routinely-collected Data (RECORD) Reporting Guidelines.

Data are available from https://opendata.nhsbsa.net/datas et/english-prescribing-data-epd. Patients were not involved in the design, conduct, reporting, or dissemination of this research, but future studies plan to conduct impact assessments on patient's lived experiences.

Ethical approval was not required for this anonymous database study and no patient identifiable data was accessed. Study was conducted in accordance with the principles set forth in the Declaration of Helsinki. There was no public and patient involvement.

\section{Results}

No known shortages were detected via formal sources, but anecdotal evidence of difficulties in acquiring stock were noted through charitable organisation's websites.

\section{Analysis by quantity}

Descriptive statistics in Table 1 show that in the pre-pandemic period, the mean and confidence intervals were higher than after the pandemic started in March-20. See Supplementary 4 for detailed total quantities by product including all clinically used agents that are not specifically discussed here e.g., mycophenolate, rapamune. The standard 
deviations in the period after March-20 have increased substantially, demonstrating wide variance in clinical practice while all confidence interval bounds are lower. The number of patients needing these therapies have been relatively stable across this timeframe as transplant services have continued for these patients with minimal disruption.

Analysis of variance (ANOVA) between the pre and post periods shows statistically significant variations with $1^{\circ}$ of freedom (1df): tacrolimus $F$ 7.432, $p=0.012$, ciclosporin $F$ $33.147, \mathrm{p}<0.001$ and sirolimus $\mathrm{F} 18.596, \mathrm{p}<0.001$. A t-test for equality of means between months (with $25 \mathrm{df}$ ) shows statistically significant mean differences for all three medicines: $71,987$ tacrolimus (95\% CI: 17,604,126,370, $\mathrm{p}=0.012)$, 59,782 ciclosporin (95\% CI:38,397,81,168, $\mathrm{p}=0.001), 3366$ sirolimus (95\% CI:1759,4974, p <0.001).

\section{Interrupted time series (ARIMA Modelling)}

The ARIMA model detailed in Table 2, shows that since March 2020, prescription volumes for these medicines have been steadily declining (see Supplementary 1 Sensitivity Analysis). The observed data points (red line) are much more dramatic than the fitted points (blue line) that our model would generate.

Examining the tacrolimus ARIMA model (\& Table 2), we can see that in the pre-pandemic period, this was growing prescription items per month at an estimated 711 doses/month. Which was accompanied by a large issuance of 233,041 doses at the interrupt point, which is then followed by monthly estimated decline of 14,524 doses in the subsequent period.
The step change is a significant change to the overall trend. The confidence intervals are both negative after the onset of the pandemic which confirms that this is an overall decrease, but with greater variation, as compared to the pre-pandemic period. Similarly, ciclosporin was already observed to be declining quite significantly in the pre-pandemic period, which seems to have slowed in the post- period but remains negative. Sirolimus shows similar terms of rapidly declining use.

Using the model 'Natural Logarithm ARIMA(1,0,0)', percentage change can be estimated (see Sensitivity analysis in Supplementary 2). It presents tacrolimus at the intercept point to be a jump of $3758 \%(p=0.018)$, followed by a deceleration of $188 \%(p=0.008)$. Similarly, ciclosporin was already declining in use prior to the pandemic by $100 \%$ $(p=0.011)$. Sirolimus at the intercept point shows a jump of $4319 \%(p=0.017)$, followed by a deceleration of $257 \%$ $(p=0.002)$. However, caution must be exercised when interpreting these estimates [25].

\section{Price analysis}

Price analysis compares the period (Jan19-Mar20) to the subsequent period (Apr20-Feb21) because of missing data on prices for March 2021. 'Actual cost' data is provided for all the months since inception of this dataset, apart from March 2021, which represents 1500 missing rows of data, data imputation was decided against because of potentially misleading analysis, in-case prices paid had increased.

Price analysis of the two timeframes shows statistically significant differences by ANOVA: tacrolimus F 8.003,
Table 1 Descriptive statistics for the monthly total quantities of prescribed medicines, standard deviation (SD), 95\% Lower and Upper confidence intervals (LCI, UCI)

\begin{tabular}{llllrrr}
\hline Period (pre, post) & Medicine & Months (N) & Mean & SD & 95\% LCI & 95\% UCI \\
\hline Jan19-Mar20 & Tacrolimus & 15 & 897,040 & 47,047 & 870,986 & 923,093 \\
Apr20-Mar21 & Tacrolimus & 12 & 825,052 & 88,019 & 769,128 & 880,977 \\
Jan19-Mar20 & Ciclosporin & 15 & 342,066 & 22,001 & 329,882 & 354,250 \\
Apr20-Mar21 & Ciclosporin & 12 & 282,284 & 31,899 & 262,016 & 302,551 \\
Jan19-Mar20 & Sirolimus & 15 & 22,880 & 1,508 & 22,045 & 23,715 \\
Apr20-Mar21 & Sirolimus & 12 & 19,514 & 2,518 & 17,914 & 21,114 \\
\hline
\end{tabular}

Table 2 Total Quantities AutoRegressive Integrated Moving Average (ARIMA) Model $(1,0,0)$, No Transformation, Autoregressive (AR) Lag 1. 'Time Period' relates to the 'Pre-pandemic period' (Jan19-Mar20), 'Phase' relating to the step-change at the interrupt time point and 'Interact' representing the (April2020-Mar2021) period

\begin{tabular}{lllll}
\hline & Column1 & Estimate & p-value & 95\% CI \\
\hline Tacrolimus-Model_1 & Time Period/Pre-pandemic period & 711 & 0.812 & $-5080,6501$ \\
Tacrolimus-Model_1 & Phase/Step-change at interrupt point & 233,041 & 0.022 & $46,989,419,092$ \\
Tacrolimus-Model_1 & Interact/Post-pandemic period & $-14,524$ & 0.008 & $-24,362,-4686$ \\
Ciclosporin-Model_2 & Time Period/Pre-pandemic period & $-3,354$ & 0.002 & $-5174,-1534$ \\
Ciclosporin-Model_2 & Phase/Step-change at interrupt point & 32,351 & 0.291 & $-26,205,90,907$ \\
Ciclosporin-Model_2 & Interact/Post-pandemic period & -2161 & 0.185 & $-5255,933$ \\
Sirolimus-Model_3 & Time Period/Pre-pandemic period & 60 & 0.465 & $-99,219$ \\
Sirolimus-Model_3 & Phase/Step-change at interrupt point & 6,291 & 0.024 & $1202,11,380$ \\
Sirolimus-Model_3 & Interact/Post-pandemic period & -485 & 0.002 & $-755,-216$ \\
\hline
\end{tabular}


p-value $=0.009$, ciclosporin F 37.584, p $<0.001$, sirolimus F 18.662, $p<0.001$ with $1 \mathrm{df}$ with significantly lower prices afterwards. A declining trend and a t-test for equality of means (with 24df) between months shows statistically significant mean differences on a monthly basis for all three medicines: tacrolimus $£ 122,995,95 \%$ CI: $£ 33,26-£ 212,730$, $\mathrm{p}=0.009$, ciclosporin $£ 76,750,95 \%$ CI: $£ 50,911-£ 102,588$, $\mathrm{p}<0.001$ and sirolimus $£ 11,484,95 \%$ CI: $£ 5,998-£ 16,971$, $\mathrm{p}<0.001$.

\section{Interrupted time series (ARIMA Modelling)}

The ARIMA model detailed in Table 3, shows that since March 2020, actual price of prescription medicines have declined (see Supplementary 2 Sensitivity Analysis). Prices are less sensitive to volume changes but demonstrate a structural down-shift tracking the lower volumes being dispensed.

Regional analysis (See Supplementary 3) show stable figures until October 2020. After this period, there are declines in the North of England, while rates in the south of England and London begin to rise. This could be due to regional lockdowns imposed in Manchester[26] and Liverpool [27] over Oct and Nov-20. National trends in the early part of the pandemic are stable. The North of England has the highest use, followed by Midlands and East of England, then South of England and finally London. As a result, we can assume that more transplant patients live in the North as compared to other parts. Oscillations are beginning to appear around November 2020 onwards: In the North, this trend is downwards. In the Midlands there is a slight spike followed by a downward trend. The South of England and London almost follow identical trends of a stable supply and then a sudden jump in the use of medications in March 2021.

Changes in the population[28-30] are minimal due to the restrictions on public travel and movement, which has been acknowledged in prior publications [18, 19]. Extrapolating provisional estimates (Birth 580,546, Death 760,020 Net $-179,474)$ gives a net decline of 180,000 in England and Wales (Population mid-2020[29] in England was 56,550,000 and in Wales was 3,170,000, equating to approximately
$59,720,000)$. Hence, the 5000 active patients on waiting lists and those who have already received a transplant were assumed to be constant because of the small variations expected in this cohort. While mortality maybe higher for these patients, no quantifiable data currently exists.

\section{Discussion}

Findings show that tacrolimus, ciclosporin and sirolimus use is declining after the onset of the pandemic, which is concerning for this vulnerable patient group. Statistically significant decline in the use of these medicines has been observed in Apr20-Mar21 as compared to Jan19-Mar20. This was however accompanied with a jump or step change at March 2020, were large quantities of medicines were issued to patients. However, this analysis suggests that that may not last long enough and is highly variable to be (un)convincing that adequate access to essential medicines[31] exists.

Price analysis shows a reduction that is unlikely to be because of better disease control but represents patients not using treatment for a variety of reasons. Similarly, regional analysis could be tracking lockdowns as they are imposed and lifted. Some artefacts could be because of regional lockdown imposed in the North of England and parts of Midlands. It also shows that as lockdown is lifted gradually in February 2021, demand returns. Important dates include 23rd March 2020 (First lockdown), 31 st October 2020 (Second lockdown), 5th January 2021 (Third lockdown) and public vaccination began in January 2021. This data contains artefacts hinting at these events.

Implications of clinical practice from our findings suggest that patients are not taking their medicines as intended by their clinical care team eg nephrologists. This may be for a variety of factors and will not be restricted to a simple notion that patients are misadherent with their therapy. It is also important to understand that this reduced prescription volume is not an indicator of fewer patients or a reduced need for these medicines. In fact, this represents a potentially growing or accelerating unmet medical need in patients who
Table 3 Total Price

ARIMA Model $(1,0,0)$, No

Transformation, Autoregressive

(AR) Lag 1. 'Time Period'

relates to the 'Pre-pandemic period' (Jan19-Mar20), 'Phase' relating to the step-change at the interrupt time point and 'Interact' representing the April2020-Feb2021 period

\begin{tabular}{lllrll}
\hline Medicine & Period & Parameter Estimate & p-value & $95 \%$ LCI & 95\% UCI \\
\hline Tacrolimus-Model_1 & TimePeriod & $-£ 246$ & 0.960 & $-£ 9,795$ & $£ 9,302$ \\
Tacrolimus-Model_1 & Phase & $£ 380,070$ & $\mathbf{0 . 0 4 1}$ & $£ 38,091$ & $£ 722,050$ \\
Tacrolimus-Model_1 & Interact & $-£ 23,280$ & 0.022 & $-£ 41,759$ & $-£ 4,802$ \\
Ciclosporin-Model_2 & TimePeriod & $-£ 4,165$ & $<0.001$ & $-£ 6,053$ & $-£ 2,278$ \\
Ciclosporin-Model_2 & Phase & $£ 27,071$ & 0.440 & $-£ 40,342$ & $£ 94,484$ \\
Ciclosporin-Model_2 & Interact & $-£ 2,201$ & 0.244 & $-£ 5,800$ & $£ 1,399$ \\
Sirolimus-Model_3 & TimePeriod & $£ 139$ & 0.622 & $-£ 407$ & $£ 686$ \\
Sirolimus-Model_3 & Phase & $£ 19,632$ & 0.059 & $£ 370$ & $£ 38,895$ \\
Sirolimus-Model_3 & Interact & $-£ 1,542$ & $\mathbf{0 . 0 0 8}$ & $-£ 2,572$ & $-£ 512$ \\
\hline
\end{tabular}

Bold text in Table 3 is drawing the reader's attention to statistically-significant values 
should be engaging with healthcare services and proactively seeking care but are not doing so with the potential (and real) risk of damage to their health. It could imply future organ rejections at higher rates with more complications or failures than expected.

The national rate of graft survival five years after first adult living donor kidney only transplant is $93 \%$ [32]. This helps generate a hypothesis that five-year organ survival rates may fall below this rate, which currently stands at risk-adjusted percentage ranging from 88 to $97 \%$ between hospitals that are national kidney transplant centres. The same 2019-2020 report suggests that Manchester has the largest proportion of patients on the waiting list and the suspended transplant list, with 10-year risk-adjusted survival rates, showing that Leicester, Newcastle and Manchester fell below $99.8 \%$ lower confidence limit, suggesting that 10 -year survival for these patients may be significantly lower than the national rate. This information accompanied with our findings suggest that these patients will be at an even higher risk than expected by 'NHS Blood and Transplant' because of patients foregoing treatment as detected in this analysis, which could perpetuate health inequities along ethnic lines as these areas have a higher percentage of Black and Asian ethnic minority populations. From the introduction, we know that ethnic minority patients wait longer for a kidney donation, face more barriers and personal stigma due to cultural differences and are independently more at risk of getting and dying from COVID due to a variety of revealed health inequalitys and care disparities experienced by this segment of the society.

To some extent, this suggestion is bearing true already from the following NHS quote: "The Organ and Tissue Donation and Transplantation Annual Activity Report 2020/21 shows that despite the global pandemic, levels were sustained at $75 \%$ of normal deceased donation activity and around $80 \%$ of normal transplant activity. Patients on the active waiting list for a transplant in the UK fell to 4,256 at the end of March 2021, however, this does not fully reflect the number of people who need an organ transplant as any patients were removed from the transplant list or transplant programmes closed during the peak of the pandemic as it was riskier to carry out transplants and NHS resources were under extra pressure. About 487 patients died waiting last year, compared with 372 the previous year. Transplant centres are still getting through the backlog of new patient referrals and are currently re-activating those who were temporarily suspended. It is currently forecast that the number of those likely waiting for a transplant in the UK is now around 7,000 - a figure last seen in 2012/13."[33]

This raises important supply or access barriers to care and places a moral and ethical concern over the donors and the donees health. One of the potential underlying reasons may relate to the structural service pathways of prescription generation and issuance of medication. This pandemic has highlighted that suspension of face-to-face care by clinicians in primary care has transferred this prescription request/supply pathway to a variety of other providers (e.g., pharmacies, 111 ), and potentially via pathways that patients find difficult to navigate especially if English is not their primary language. It maybe the inflexibility of the primary healthcare interface which means it is not as responsive as needed during a natural disaster.

The number of people on transplant in the UK are the highest in Europe and the care needs they embody are not marginal and do not resolve quickly. These patients may have occasion for repeat episodes of needing kidney care over their life course too. Health demands like these, bring problems of comorbidities, financial insecurity, mental health concerns and a variety of other health and pandemic related pressures that patients may be facing.

So, it is vitally important to build a fit for purpose service that is flexible and responsive, even during unprecedented pressures that puts the onus of care on clinicians rather than patient. Patient tracing and follow-up is possibly the way forward in ensuring that care gaps do not further escalate as demonstrated with the North of England, leading to a postcode lottery and further entrenching a north versus south divide. These patients will be suffering and may not be able to seek care, with consequent suffering and potentially higher rates of mortality. This study brings an early warning signal and provides opportunity for clinicians to follow up their patient-lists, especially those patients who have not been seen in a long while.

Normally, these patients are motivated to maintain their health and well-being and take their medicines as prescribed and intended lack of adherence is not really an issue. Given this, and study findings, it is hypothesised that the data maybe detecting a structural problem in accessing medicines. further work needs to appraise these patients and lived experiences of the pandemic qualitatively and quantitatively. Patients need to be invited to participate in co-constructed research to consider how service architecture and delivery, needs to be redesigned to become accessible.

It can be marginally argued that the limitations of this study prevent absolute confirmation of these claims. However, clinicians can draw on their own individual experiences during the pandemic to see whether these claims fit with their lived experiences. This paper seeks to provide the research evidence to be able to make better patient-centred, evidence-based, care decisions in a rapidly changing healthcare environment.

Strengths include data being rooted in national level statistics representing a universal healthcare system and how it has coped during the pandemic. Limitations may pertain to the completeness and accuracy of the governmental data. Study analysis is restricted to England and findings may only 
be generalisable to a limited extent to the devolved nations of Wales, Scotland and Northern Ireland or other countries, including in Europe.

\section{Conclusions}

In a large national cohort of patients tacrolimus, ciclosporin and sirolimus use is declining after the onset of the pandemic, which shows a reduction that is unlikely to be because of better disease control but represents patients not getting treatment representing growing unmet medical need. Patient tracing and urgent follow-up is recommended.

A video (11 min) briefly explaining the study methods, results, the implications to clinical practise: https://brighton. cloud.panopto.eu/Panopto/Pages/Viewer.aspx?id=540d6 084-c1ae-4f84-8835-ad4f00c3d1f3

Supplementary Information The online version contains supplementary material available at https://doi.org/10.1007/s40199-021-00431-7.

Authors contributions The authors conceived, designed the work; conducted the analysis, interpreted the data; drafted the work, revised it critically for important intellectual content; approved the version to be published; and agree to be accountable for all aspects of the work, meeting ICMJE criteria as the Author.

Funding This study was funded by the University of Brighton. The sponsor has not been directly involved in the research.

Data availability The data supporting the findings of this study are openly available at: https://opendata.nhsbsa.net/dataset/english-presc ribing-data-epd

Video summary $(11 \mathrm{~min})$ briefly explaining the study methods, results, the implications to clinical practise: https://brighton.cloud. panopto.eu/Panopto/Pages/Viewer.aspx?id=540d6084-c1ae-4f84$8835-a d 4 f 00 c 3 d 1 f 3$

\section{Declarations}

Competing interests The authors have no competing interests to declare that are relevant to the content of this article.

Ethical approval Ethical approval was not required for this anonymous database study, which analysed freely and publicly available government data under the Open Government Licence (OGL) v2.0. The study was conducted according to the Declaration of Helsinki.

\section{References}

1. Neuwirt H, Rudnicki M, Schratzberger P, Pirklbauer M, Kronbichler A, Mayer G. Immunosuppression after renal transplantation memo. 2019;12:216-21.

2. Medicines and Healthcare products Regulatory Agency. Oral tacrolimus products: reminder to prescribe and dispense by brand name only [Internet]. GOV.UK. [cited 2020 May 28].
Available from: https://www.gov.uk/drug-safety-update/ oral-tacrolimus-products-reminder-to-prescribe-and-dispe nse-by-brand-name-only

3. Immunosuppressive therapy for kidney transplant in adults; Technology appraisal guidance [TA481] [Internet]. National Institute for Health and Care Excellence (UK); 2017 [cited 2021 Jun 22]. Available from: https://www.nice.org.uk/guidance/ta481

4. Karpe KM, Talaulikar GS, Walters GD. Calcineurin inhibitor withdrawal or tapering for kidney transplant recipients. Cochrane Database Syst Rev. 2017;7:CD006750.

5. Immunosuppressants: COVID-19 respiratory infections: 16 case reports. Reactions Weekly. 2021;1855:183-183.

6. Abu Jawdeh BG. COVID-19 in Kidney Transplantation: Outcomes, Immunosuppression Management, and Operational Challenges. Adv Chronic Kidney Dis. 2020;27:383-9.

7. Ahmed AR, Ebad CA, Stoneman S, Satti MM, Conlon PJ. Kidney injury in COVID-19. WJN. 2020;9:18-32.

8. Kellum JA, van Till JWO, Mulligan G. Targeting acute kidney injury in COVID-19. Nephrol Dial Transplant. 2020;35:1652-62.

9. COVID-19 and Kidney Disease: Update on Epidemiology, Clinical Manifestations, Pathophysiology and Management. J Coll Physicians Surg Pak. 2020;30:19-25.

10. Couch J, Liddy N, McDougall J. 'Our Voices Aren't in Lockdown'-Refugee Young People, Challenges, and Innovation During COVID-19. JAYS [Internet]. 2021 [cited 2021 Jun 22]; Available from: https://link.springer.com/https://doi.org/10.1007/ s43151-021-00043-7

11. DeFilippis EM, Reza N, Donald E, Givertz MM, Lindenfeld J, Jessup M. Considerations for Heart Failure Care During the COVID19 Pandemic. JACC: Heart Failure. 2020;8:681-91.

12. Ku E, Lee BK, McCulloch CE, Roll GR, Grimes B, Adey D, et al. Racial and Ethnic Disparities in Kidney Transplant Access Within a Theoretical Context of Medical Eligibility. Transplantation. 2020;104:1437-44.

13. Grossi AA, Maggiore U, Puoti F, Grossi PA, Picozzi M, Cardillo M. Association of immigration background with kidney graft function in a publicly funded health system: a nationwide retrospective cohort study in Italy. Transpl Int. 2020;33:1405-16.

14. Pankhurst T, Evison F, Mytton J, Williamson S, Kerecuk L, Lipkin G. Young adults have worse kidney transplant outcomes than other age groups. Nephrol Dial Transplant. 2020;35:1043-51.

15. Transplant centre closures and restrictions [Internet]. ODT Clinical - NHS Blood and Transplant. [cited 2020 May 25]. Available from: https://www.odt.nhs.uk/deceased-donation/covid-19advice-for-clinicians/transplant-centre-closures-and-restrictions/

16. Barrett R. Reduced prescription tacrolimus use: a cross-sectional analysis of England's national prescription statistics during the COVID-19 pandemic, by region. Kidney Int. 2020;98:778-9.

17. Institute for Government analysis. Timeline of UK coronavirus lockdowns, March 2020 to March 2021 [Internet]. [cited 2021 Jun 22]. Available from: https://www.instituteforgovernment.org.uk/ sites/default/files/timeline-lockdown-web.pdf

18. Barrett R, Barrett R, Dhar K, Birch B. Gonadorelins adherence in prostate cancer: A time-series analysis of England's national prescriptions during the COVID-19 pandemic (Jan 2019 to Oct 2020). BJUI Compass [Internet]. 2021; Available from: https:// doi.org/10.1111/bco2.101

19. Barrett R, Barrett R. Asthma and COPD medicines prescriptionclaims: A time-series analysis of England's national prescriptions during the COVID-19 pandemic (Jan 2019 to Oct 2020). Expert Rev Respir Med. 2022;2021(17476348):1985470.

20. English Prescribing Dataset (EPD) - Open Data Portal BETA [Internet]. [cited 2020 Apr 25]. Available from: https://opendata. nhsbsa.net/dataset/english-prescribing-data-epd

21. Cochrane Effective Practice and Organisation of Care (EPOC). Interrupted time series (ITS) analyses. EPOC Resources for 
review authors [Internet]. 2017. Available from: epoc.cochrane. org/resources/epoc-specific-resources-review-authors

22. Lopez Bernal J, Cummins S, Gasparrini A. Interrupted time series regression for the evaluation of public health interventions: a tutorial. Int J Epidemiol. 2016; dyw098.

23. Kontopantelis E, Doran T, Springate DA, Buchan I, Reeves D. Regression based quasi-experimental approach when randomisation is not an option: interrupted time series analysis. BMJ. 2015;350:h2750-h2750.

24. Sato RC. Disease management with ARIMA model in time series. Einstein (Sao Paulo). 2013;11:128-31.

25. Benoit K. Linear regression models with logarithmic transformations. London School of Economics. 2011;22:23-36.

26. Covid: Greater Manchester facing "winter of hardship" without support - Burnham. BBC News [Internet]. 2020 Oct 20 [cited 2021 Nov 26]; Available from: https://www.bbc.com/news/uk54617898

27. Ellyatt H. "It's going to be a very tough six months": Strict lockdown in Liverpool leads to fear and anger [Internet]. CNBC. 2020 [cited 2021 Nov 26]. Available from: https://www.cnbc.com/2020/ 10/14/liverpool-lockdown-leads-to-backlash.html

28. Office for National Statistics. Vital statistics in the UK: births, deaths and marriages [Internet]. 2021 [cited 2021 Mar 19]. Available from: https://www.ons.gov.uk/peoplepopulationandcommun ity/populationandmigration/populationestimates/datasets/vitalstati sticspopulationandhealthreferencetables

29. Office for National Statistics. Population estimates for the UK, England and Wales, Scotland and Northern Ireland [Internet].
2021 [cited 2021 Oct 24]. Available from: https://www.ons.gov. uk/peoplepopulationandcommunity/populationandmigration/ populationestimates/bulletins/annualmidyearpopulationestimat es/mid2020\#age-structure-of-the-uk-population

30. Office for National Statistics. Overview of the UK population [Internet]. [cited 2021 Nov 25]. Available from: https://www. ons.gov.uk/peoplepopulationandcommunity/populationandmigrati on/populationestimates/articles/overviewoftheukpopulation/janua ry2021

31. World Health Organization. WHO Model Lists of Essential Medicines [Internet]. [cited 2021 Oct 9]. Available from: https://www. who.int/groups/expert-committee-on-selection-and-use-of-essen tial-medicines/essential-medicines-lists

32. NHS Blood and Transplant. Annual Report on Kidney Transplantation 2019/20 [Internet]. 2020. Available from: https://nhsbt dbe.blob.core.windows.net/umbraco-assets-corp/20032/kidneyannual-report-2019-20-final.pdf

33. NHS Organ Donation. Organ donation achieved three quarters of its normal activity last year despite global pandemic [Internet]. NHS Organ Donation. 2021 [cited 2021 Nov 26]. Available from: https://www.organdonation.nhs.uk/get-involved/news/organ-donat ion-achieved-three-quarters-of-its-normal-activity-last-year-despi te-global-pandemic/

Publisher's note Springer Nature remains neutral with regard to jurisdictional claims in published maps and institutional affiliations.

\section{Authors and Affiliations}

\section{Ravina Barrett ${ }^{1}[0$}

Ravina Barrett

R.Barrett2@Brighton.ac.uk

1 School of Applied Sciences, University of Brighton, Cockcroft Building, Moulsecoomb, Brighton BN2 4GJ, England 